\title{
Brain neurotransmitter transporter/receptor genomics and efavirenz central nervous system adverse events David W. Haas ${ }^{\mathrm{a}, \mathrm{b}}$, Yuki Bradford ${ }^{\mathrm{c}}$, Anurag Verma ${ }^{\mathrm{c}, \mathrm{d}}$, Shefali S. Verma ${ }^{\mathrm{c}}$, Joseph J. Eron ${ }^{\text {h }}$, Roy M. Gulick ${ }^{f}$, Sharon A. Riddler ${ }^{\text {e }}$, Paul E. Sax', Eric S. Daar', Gene D. Morse ${ }^{\mathrm{g}}$, Edward P. Acosta ${ }^{k}$ and Marylyn D. Ritchie ${ }^{\mathrm{c}, \mathrm{d}}$
}

Objective We characterized associations between central nervous system (CNS) adverse events and brain neurotransmitter transporter/receptor genomics among participants randomized to efavirenz-containing regimens in AIDS Clinical Trials Group studies in the USA.

Participants and methods Four clinical trials randomly assigned treatment-naive participants to efavirenzcontaining regimens. Genome-wide genotype and PrediXcan were used to infer gene expression levels in tissues including 10 brain regions. Multivariable regression models stratified by race/ethnicity were adjusted for CYP2B6/CYP2A6 genotypes that predict plasma efavirenz exposure, age, and sex. Combined analyses also adjusted for genetic ancestry.

Results Analyses included 167 cases with grade 2 or greater efavirenz-consistent CNS adverse events within 48 weeks of study entry, and 653 efavirenz-tolerant controls. CYP2B6/CYP2A6 genotype level was independently associated with CNS adverse events (odds ratio: $1.07 ; P=0.044)$. Predicted expression of six genes postulated to mediate efavirenz CNS side effects (SLC6A2, SLC6A3, PGR, HTR2A, HTR2B, HTR6) were not associated with CNS adverse events after correcting for multiple testing, the lowest $P$ value being for $P G R$ in hippocampus $(P=0.012)$, nor were polymorphisms in these genes or $A R$ and $H T R 2 C$, the lowest $P$ value being for rs12393326 in HTR2C $\left(P=6.7 \times 10^{-4}\right)$. As a positive control, baseline plasma bilirubin concentration was associated with predicted liver UGT1A1 expression level $\left(P=1.9 \times 10^{-27}\right)$.

Conclusion Efavirenz-related CNS adverse events were not associated with predicted neurotransmitter transporter/ receptor gene expression levels in brain or with polymorphisms in these genes. Variable susceptibility to efavirenz-related CNS adverse events may not be explained by brain neurotransmitter transporter/receptor genomics. Pharmacogenetics and Genomics 28:179-187 Copyright (c) 2018 Wolters Kluwer Health, Inc. All rights reserved.

Pharmacogenetics and Genomics 2018, 28:179-187

Keywords: efavirenz, HIV, neurotransmitter receptor, neurotransmitter transporter, pharmacogenomics

${ }^{a}$ Department of Medicine, Vanderbilt University School of Medicine, ${ }^{b}$ Department of Internal Medicine, Meharry Medical College, Nashville, Tennessee, 'Department of Genetics, Institute for Biomedical Informatics, University of Pennsylvania, Perelman School of Medicine, Philadelphia, ${ }^{e}$ Department of Medicine, University of Pittsburgh, Pittsburgh, Pennsylvania, 'Department of Medicine, Weill Cornell Medicine, New York City, ${ }^{9}$ University at Buffalo, SUNY, Buffalo, New York,

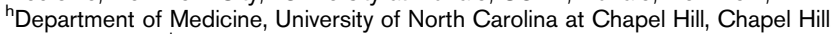
North Carolina, 'Department of Medicine, Brigham and Women's Hospital and Harvard Medical School, Boston, Massachusetts, 'David Geffen School of Medicine at UCLA, Harbor-UCLA Medical Center, Los Angeles Biomedical Research Institute, Los Angeles, California and 'University of Alabama at Birmingham, Birmingham, Alabama, USA

Correspondence to David W. Haas, MD, Vanderbilt Health, One Hundred Oaks, 719 Thompson Lane, Suite 47183, Nashville, TN 37204, USA

Tel: + 1615936 8594; fax: + 16159362644 ;

e-mail: david.haas@vanderbilt.edu

Received 11 December 2017 Accepted 10 May 2018

present with CYP2B6 slow metabolizer genotypes $[19,22]$. These polymorphisms explain $\sim 35 \%$ of interindividual variability in plasma efavirenz exposure [15]. A possible association with efavirenz pharmacokinetics has also been reported with $U G T 2 B 7$ genotype [21], but with small effect size [22].

Increased likelihood of efavirenz CNS symptoms has been attributed to $C Y P 2 B 6$ slow metabolizer genotypes $[18,23,24]$. In an initial analysis of AIDS Clinical Trials Group (ACTG) data [18], CYP2B6 slow metabolizer genotypes were associated with adverse events in 276 White participants $(P=0.04)$, but not in 217 Black par-

ticipants $(P=0.58)$. Similarly, among 563 patients who
Supplemental Digital Content is available for this article. Direct URL citations appear in the printed text and are provided in the HTML and PDF versions of this article on the journal's website, www.pharmacogeneticsandgenomics.com. 
initiated efavirenz-containing regimens at a clinic in the Southeastern USA, slow metabolizer CYP2B6 genotypes were associated with efavirenz discontinuation for CNS symptoms in 335 White patients $(P=0.001)$, but not in 198 Black patients $(P=0.27)$ [23]. Among 1833 ACTG study participants in the USA, an association between CYP2B6 genotype and suicidality was strongest among White participants, but nearly null among Black participants [24]. The reason for this apparent difference by race is not known.

Brain neurotransmitter transporters/receptors are postulated to mediate efavirenz CNS symptoms, including the norepinephrine transporter (encoded by $S L C 6 A 2$ ), dopamine transporter $(S L C 6 A 3)$, progesterone PR-B $(P G R)$, serotonin receptors 5-HT2A (HTR2A), 5-HT2B (HTR2B), 5-HT2C (HTR2C), and 5-HT6 (HTRO), and androgen receptor (AR) (Daria Hazuda, personal communication, 1st March 2016). Lower expression levels of neurotransmitter transporter/ receptor genes in brain tissue could possibly confer increased susceptibility to efavirenz side effects. This hypothesis may be addressed indirectly using, PrediXcan, a novel computational algorithm that uses genome-wide genotype data to infer RNA expression levels for individual genes in various human organs and tissues [25].

We examined whether predicted expression levels of selected neurotransmitter transporter/receptor genes in brain were associated with risk for CNS adverse events in participants randomized to receive efavirenz-containing regimens in ACTG studies. We also examined whether individual polymorphisms in these genes were associated with CNS adverse events with efavirenz.

\section{Participants and methods Study design and participants}

Data were pooled from antiretroviral-naive individuals who had been randomly assigned to initiate efavirenzcontaining regimens in four studies: ACTG384 (ClinicalTrials. gov: NCT00000919) [26,27], A5095 (ClinicalTrials.gov: NGT00013520) [2,28], A5142 (NCT00050895) [3], and A5202 (NCT00118898) [6]. Drug class components of the regimens were randomly assigned (efavirenz-based regimen vs. comparator regimen) except for nucleoside analog choice in A5142. Genetic association testing was limited to participants who consented to genetic testing under ACTG protocol A5128 [29]. Participants self-reported race/ethnicity.

Each protocol required reporting of signs, symptoms, or diagnoses at each visit, severe and life-threatening graded signs or symptoms [30], and signs or symptoms that led to change in study regimen. Diagnoses were not graded. Further, study A5142 required report of all moderate signs or symptoms, study ACTG384 required entry of all signs and symptoms grade 3 or greater, all signs and symptoms that resulted in dose modification regardless of grade, and all grade 2 or greater CNS symptoms, and A5095 and A5202 required report of moderate CNS symptoms. Site institutional review boards approved each study, and participants provided written informed consent.

\section{Outcomes}

The outcome of interest was new onset grade 2 or greater CNS signs or symptoms that were consistent with possible efavirenz effect. These included agitation, behavior changes, abnormal cognition, confusion, depression, difficulty concentrating, dizziness, abnormal dreams, excessive anger, hyperactivity, inappropriate behavior, insomnia, lethargy, change in level of consciousness, light-headedness, memory loss, psychiatric mental status change, rage, or sleeping problems. Adverse event data were based on self-report, and did not involve questionnaires specifically targeting CNS events. Death by suicide was also considered a possible efavirenz effect.

Cases were participants with grade 2 or greater efavirenzconsistent CNS signs or symptoms, or with death due to suicide, documented within 48 weeks after study entry while still being prescribed efavirenz, or within 2 weeks after efavirenz was discontinued. Controls were participants with no documented efavirenz-consistent CNS signs or symptoms regardless of any grade after study entry while being prescribed efavirenz for at least 96 weeks. Cases and controls were excluded for any neuropsychological signs or symptoms of any grade documented at study entry.

\section{Covariates}

Baseline covariates included in multivariable models included age, sex, and CYP2B6/CYP2A6 genotype. The first two principal components generated from genome-wide genotype data were also included to minimize confounding by unrecognized population stratification. Analyses performed separately among self-identified White, Black, and Hispanic participants also adjusted for age, sex, and CYP2B6/CYP2A6 genotype, but not principal components.

\section{Genetic assays and data}

Genotypes for CYP2B6 516G $\rightarrow \mathrm{T}, 983 \mathrm{~T} \rightarrow \mathrm{C}, 15582 \mathrm{C} \rightarrow \mathrm{T}$, and CYP2A6 $-48 \mathrm{~T} \rightarrow \mathrm{G}$ were largely available from a MassARRAY iPLEX Gold (Sequenom Inc., San Diego, California, USA) assay, generated by Vanderbilt Technologies for Advanced Genomics [15]. Genome-wide genotype data largely available from a previous immunogenomics project [31] were generated by Illumina HumanHap 650Y array for A5095 and by Illumina 1M duo array for A5142 and A5202 (Illumina, San Diego, California, USA). Quality control and imputation of genome-wide data were performed as described elsewhere [32]. The PLINK program and R statistical programming language were used for QC procedures [33,34]. Polymorphisms were censored for call rates of less than $99 \%$. We excluded 18 samples where genetically inferred sex differed from clinical data, or missing sex status that could not be inferred, 105 samples with overall genotyping call rates less than $99 \%$, and 17 samples with cryptic relatedness based on 
identity by descent estimates more than 0.3 from $\sim 100000$ pruned single nucleotide polymorphisms (SNPs).

Post-QC data were imputed to 1000 Genomes [35] after converting to genome build 37 using liftOver [36] and stratifying by chromosome to parallelize imputation processing. ShapeIt2 [37] was used to check strand alignment and to phase data. The IMPUTE2 algorithm [38] was used to impute additional genotypes that were available in the 1000 Genomes reference panel, but not directly genotyped. Each chromosome was segmented into $6 \mathrm{Mb}$ regions with at least 3500 reference variants in each region. Imputed genotypes were included if posterior probabilities exceeded 0.9.

Quality of imputed data was assessed following the Electronic Medical Records and Genomics protocol [39]. Each chromosome from each phase was checked for $100 \%$ concordance with genotyped data. We dropped imputed SNPs with info scores of less than 0.7, genotyping call rates of less than $99 \%$ and minor allele frequencies of less than 0.01 .

Twelve composite CYP2B6/CYP2A6 genotype levels that predict progressively greater plasma efavirenz exposure were defined by combinations of three CYP2B6 and one CYP2A6 polymorphisms [15,22] as described elsewhere [22]. Each CYP2B6/CYP2A6 polymorphism (rs3745274, rs28399499, rs4803419, and rs28399433) was in Hardy-Weinberg equilibrium in White, Black, and Hispanic participants analyzed separately except rs4803419 in White participants (multiple testing-corrected $P=0.045$ ). Consent for genetic analysis was obtained under ACTG protocol A5128 [29], and the ACTG approved this use of DNA.

\section{PrediXcan}

PrediXcan was used to infer, from genome-wide genotype data, the heritable component of RNA expression levels in 43 available reference tissues [25], using the 2015 PrediXcan models. PrediXcan was able to infer expression of $\sim 10000$ genes in each tissue. The 10 reference brain tissues analyzed included anterior cingulate cortex, caudate, cerebellar hemisphere, cerebellum, cortex, frontal cortex, hippocampus, hypothalamus, nucleus accumbens, and putamen. The additional 33 reference tissues included adipose subcutaneous, adrenal gland, aorta, coronary artery, tibial artery, breast, Epstein-Barr virus-transformed lymphocytes, transformed fibroblasts, sigmoid colon, transverse colon, gastroesophageal junction, esophagus mucosa, esophagus muscularis, heart atrial appendage, heart left ventricle, liver, lung, skeletal muscle, tibial nerve, ovary, pancreas, pituitary gland, skin suprapubic not sun exposed, skin lower leg sun exposed, terminal ileum, terminal ileum (elastic net), spleen, stomach, testis, thyroid, whole blood unscaled, whole blood, and cross tissue.

\section{Statistical analysis}

We characterized associations between predicted expression of six autosomal brain neurotransmitter transporter/receptor genes (SLC6A2, SLC6A3, PGR, HTR2A, HTR2B, HTR6) and efavirenz CNS adverse events. We also characterized associations between polymorphisms in these six genes as well as $A R$ and $H T R 2 C$ and efavirenz CNS adverse events. Because $A R$ and HTR2C are on the $\mathrm{X}$ chromosome, PrediXcan cannot infer their expression. Analyses controlled for CYP2B6/CYP2A6 genotype level as a linear covariate, age, and sex. The first two principal components, calculated using EIGENSOFT [40] were used to adjust for global ancestry in analyses that pooled all race/ethnicity groups. Associations with CNS adverse events were evaluated using logistic regression models, stratified by race/ethnicity. As a positive control, associations between baseline total plasma bilirubin concentration and hepatic UGT1A1 expression were similarly evaluated, controlling for CYP2B6/CYP2A6 genotype level, age, sex, and the first two principal components. The Bonferroni method was used to adjust for multiple testing.

\section{Results}

\section{Study participants}

Of 4742 participants from ACTG studies ACTG384, A5095, A5142, and A5202, a total of 2171 who had been randomly assigned to initiate efavirenz-containing regimens consented to genetic testing at research sites in the USA. Of these participants, 1425 were successfully genotyped for CYP2B6/CYP2A6 and had imputed genome-wide genotype and principal component data. Of these participants, 820 met definitions as either cases with documented grade 2 or greater efavirenz-consistent CNS adverse events by week 48 $(n=167)$ or efavirenz-tolerant controls that continued efavirenz for at least 96 weeks without documented CNS adverse events $(n=653)$. Participant disposition is shown in Fig. 1. Baseline characteristics of study participants are presented in Table 1. Females were underrepresented among cases versus controls.

\section{CYP2B6/CYP2A6 genotype and CNS adverse events}

In logistical regression analyses, among the 820 participants who were evaluable as either cases who developed grade 2 or greater CNS adverse events by week $48(n=167)$, or efavirenz-tolerant controls $(n=653)$, and controlling for age, sex, and the first two principal components, CYP2B6/CYP2A6 genotype level was associated with grade 2 or greater CNS adverse events within 48 weeks [odds ratio (OR): 1.07; 95\% confidence interval (CI): $1.00-1.15 ; P=0.044]$. In analyses performed separately among 335 White, 264 Black, and 184 Hispanic participants, and adjusted for age and sex but not principal components, ORs for association between CYP2B6/ CYP2A6 genotype level and grade 2 or greater CNS event by week 48 were similar to the total group (1.10 for White, 1.07 for Black, and 1.08 for Hispanic participants), but none were statically significant $(P>0.10$ for each). In the above multivariable models, female sex was associated with fewer grade 2 or greater CNS adverse events within 48 weeks among all participants (OR: 0.33; 95\% CI: 0.17-0.63; $P=0.001$ ), and among White participants (OR: 0.14; 95\% CI: 0.18-1.04; $P=0.055)$ and Black participants (OR: $0.43 ; 95 \%$ CI: $0.19-0.96 ; P=0.040)$ analyzed separately. 
Fig. 1

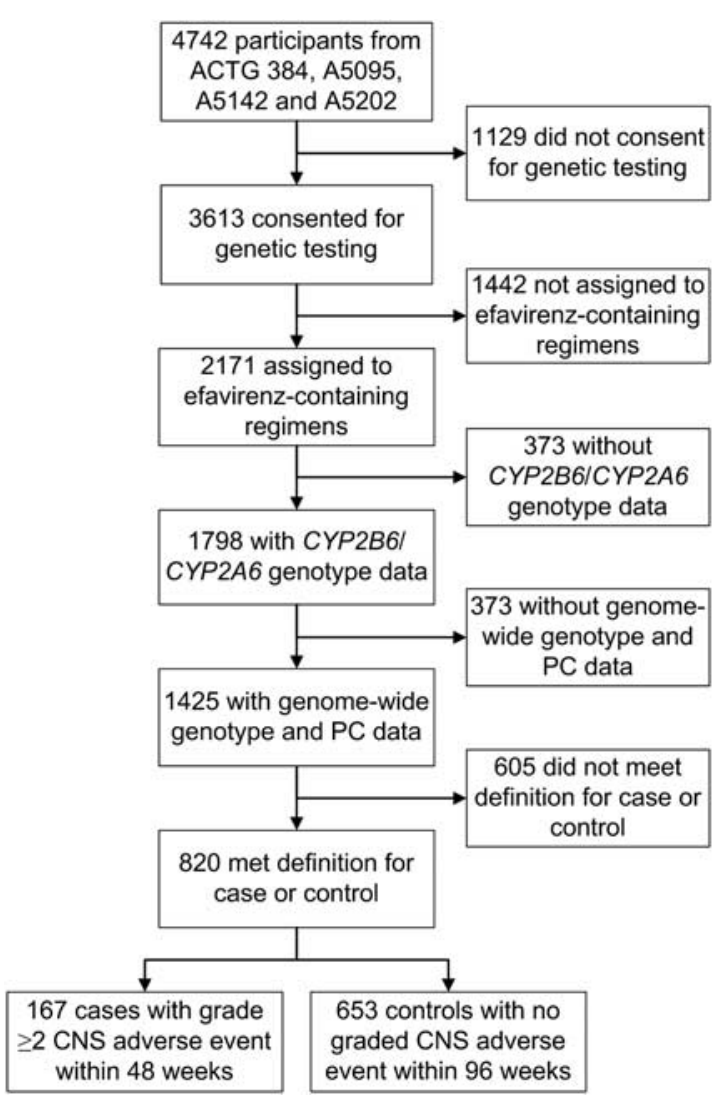

Derivation of the study sample. Derivation cases who developed grade 2 or greater efavirenz-consistent central nervous system (CNS) adverse events by week 48 , and efavirenz-tolerant controls who continued efavirenz for at least 96 weeks without efavirenz-consistent CNS adverse events, during participation in ACTG384, A5095, A5142, or A5202 are shown. PC, principal components.

Table 1 Baseline characteristics of participants included in analyses of grade 2 or greater central nervous system adverse events after being randomly assigned to efavirenz-containing regimens

\begin{tabular}{lccc}
\hline & $\begin{array}{c}\text { Cases } \\
(n=167)\end{array}$ & $\begin{array}{c}\text { Controls } \\
(n=653)\end{array}$ & $\begin{array}{c}\text { Total } \\
(N=820)\end{array}$ \\
\hline $\begin{array}{l}\text { Parent study [n (\%)] } \\
\text { ACTG384 }\end{array}$ & $28(13.9)$ & $173(86.1)$ & 201 \\
A5095 & $63(34.4)$ & $120(65.6)$ & 183 \\
A5142 & $15(10.1)$ & $133(89.9)$ & 148 \\
A5202 & $61(21.2)$ & $227(78.8)$ & 288 \\
Sex [n (\%)] & & & \\
$\quad$ Male & $156(16.4)$ & $539(77.6)$ & 695 \\
$\quad$ Female & $11(8.8)$ & $114(91.2)$ & 125 \\
Race or ethnic group [n (\%)] & & & \\
$\quad$ White non-Hispanic & $81(22.8)$ & $274(77.2)$ & 355 \\
$\quad$ Black non-Hispanic & $53(20.1)$ & $211(79.9)$ & 264 \\
$\quad$ Hispanic & $26(14.1)$ & $158(85.9)$ & 184 \\
Asian & $2(25.0)$ & $6(75.0)$ & 8 \\
Native American & $3(42.9)$ & $4(57.1)$ & 7 \\
Multiracial & $1(100)$ & $0(0)$ & 1 \\
$\quad$ Unknown & $1(100)$ & $0(0)$ & 1 \\
Age [median (IOR)] & $38(31-45)$ & $37(31-45)$ & $37(31-45)$ \\
$\quad$ (years) & & & \\
\hline
\end{tabular}

IQR, interquartile range.
Predicted expression levels of neurotransmitter transporter/receptor genes and CNS adverse events

For each participant, genome-wide genotype data were used to predict the heritable component of gene expression for 43 tissues, including multiple reference regions of the brain. Primary analyses characterized associations between six neurotransmitter transporter/receptor genes postulated to mediate efavirenz effects in brain (SLC6A2, SLC6A3, PGR, HTR2A, HTR2B, HTR6), and grade 2 or greater CNS adverse events within 48 weeks. Among all participants, and controlling for CYP2B6/CYP2A6 genotype level, age, sex, and two principal components, the lowest nominal $P$ value was for $P G R$ in hippocampus $(P=0.012)$. The two lowest nominal $P$-value results for each gene and associated brain regions are presented in Table 2. None were significant after correcting for multiple testing. In analyses performed separately among White, Black, and Hispanic participants, and adjusted for age and sex but not principal components, there were no significant associations. The lowest $P$ value in White participants was for HTR2A in cerebellar hemisphere $(P=0.12)$, in Black participants was for SLC6A2 in caudate $(P=0.17)$, and in Hispanics participants was for HTR2A in cortex $(P=0.082)$. The two lowest nominal $P$-value results for each gene within race/ethnicity group and associated brain regions are presented in Supplementary Table 1 (Supplemental digital content 1, http://links.lww.com/NMC/A133).

Following the approach of Li et al. [41], we were able to assess correlations between predicted and observed gene expressions in reference datasets in brain tissue for SLC6A3, HTR2B, and HTR6. Among Whites, the strongest correlations were with SLC6A3 $\left(r^{2}=0.038\right)$ and $H T R 2 B$ in hypothalamus $\left(r^{2}=0.028\right)$. Among Africans, the strongest correlations were with SLC6A3 in hypothalamus $\left(r^{2}=0.040\right)$

Table 2 Associations between predicted expression levels of six autosomal neurotransmitter receptor/transporter genes and grade 2 or greater central nervous system adverse events within 48 weeks of starting efavirenz-containing regimens

\begin{tabular}{|c|c|c|c|c|}
\hline Genes & Chromosome & Brain region & $\beta$ & $P$ value \\
\hline \multirow[t]{2}{*}{ HTR2A } & 13 & Cerebellar hemisphere & -0.61 & 0.086 \\
\hline & & Frontal cortex & -1.24 & 0.677 \\
\hline \multirow[t]{2}{*}{ HTR2B } & 2 & Frontal cortex & -0.77 & 0.331 \\
\hline & & Hippocampus & -0.31 & 0.601 \\
\hline \multirow[t]{2}{*}{ HTR6 } & 1 & Nucleus accumbens & 0.45 & 0.258 \\
\hline & & Caudate & 0.53 & 0.587 \\
\hline \multirow[t]{2}{*}{$P G R$} & 11 & Hippocampus & -0.91 & 0.012 \\
\hline & & Cortex & -1.00 & 0.204 \\
\hline \multirow[t]{2}{*}{$S L C 6 A 2$} & 16 & Cortex & 1.85 & 0.405 \\
\hline & & Caudate & 0.69 & 0.454 \\
\hline \multirow[t]{2}{*}{$S L C 6 A 3$} & 5 & Nucleus accumbens & 1.59 & 0.116 \\
\hline & & Hypothalamus & 0.65 & 0.192 \\
\hline
\end{tabular}

Logistic regression analysis involved 820 total participants, which included 167 grade 2 or greater CNS event cases and 653 efavirenz-tolerant controls. The analysis controlled for CYP2B6/CYP2A6 genotype level, age, sex, and the first 2 principal components. The analyses included all evaluable participants without stratification for race/ancestry. The two lowest $P$-value results are shown for six genes postulated to mediate efavirenz CNS side effects. The positive or negative $\beta$ indicates directionality of the relationship.

CNS, central nervous system. 
and HTR6 in cortex $\left(r^{2}=0.029\right)$. These would not be considered well-predicted genes.

\section{Predicted expression levels for all evaluable genes in brain and CNS adverse events}

In secondary analyses, we explored associations with expression across all evaluable autosomal genes in the brain. Among all participants, and controlling for CYP2B6/CYP2A6 genotype level, age, sex, and two principal components, the lowest nominal $P$ value was for $R C E 1$ (a metalloproteinase) in cerebellar hemisphere $\left(P=5.3 \times 10^{-8}\right)$. The two lowest nominal $P$-value results for each brain region are presented in Table 3. In analyses performed separately among White, Black, and Hispanic participants, and adjusted for age and sex but not principal components, there were no significant associations after adjusting for multiple comparisons. The lowest $P$ value in White participants was for $A C S F 3$ in anterior cingulate cortex $\left(P=9.5 \times 10^{-6}\right)$, in Black participants was for TRPC3 in cerebellar hemisphere $\left(P=1.9 \times 10^{-5}\right)$, and in Hispanics participants was for $K L K 5$ in cerebellar hemisphere $\left(P=3.0 \times 10^{-5}\right)$. The two lowest nominal $P$-value genes for each brain region each gene within race/ethnicity group are presented in Supplementary Table 2 (Supplemental digital content 1, http://links.lwew.com/NMC/A133).

\section{Predicted UGT1A1 expression in all tissues and baseline plasma bilirubin concentration}

As a positive control we considered total plasma bilirubin concentration at baseline, which should correlate inversely with UGT1A1 expression, especially in liver. By linear

Table 3 Associations between predicted expression levels of all genes in brain and grade 2 or greater central nervous system adverse events within 48 weeks of starting efavirenz-containing regimens

\begin{tabular}{|c|c|c|c|c|}
\hline Brain regions & Gene & Chromosome & $\beta$ & $P$ value \\
\hline \multirow[t]{2}{*}{ Anterior cingulate cortex } & $P A X B P 1$ & 21 & -6.73 & $1.7 \times 10^{-6}$ \\
\hline & SEMAЗG & 3 & 2.80 & $2.8 \times 10^{-6}$ \\
\hline \multirow[t]{2}{*}{ Caudate } & EMC10 & 19 & 2.79 & $1.0 \times 10^{-5}$ \\
\hline & SIRPB1 & 20 & 1.52 & $1.9 \times 10^{-5}$ \\
\hline \multirow[t]{2}{*}{ Cerebellar hemisphere } & RCE1 & 11 & -2.64 & $5.3 \times 10^{-8}$ \\
\hline & $R A B E P K$ & 9 & -1.21 & $4.6 \times 10^{-6}$ \\
\hline \multirow[t]{2}{*}{ Cerebellum } & $C D K 10$ & 16 & -0.73 & $1.8 \times 10^{-5}$ \\
\hline & C4orf48 & 4 & -4.90 & $4.9 \times 10^{-5}$ \\
\hline \multirow[t]{2}{*}{ Cortex } & TFAP2D & 6 & -6.61 & $1.2 \times 10^{-6}$ \\
\hline & EHMT1 & 9 & -1.44 & $1.1 \times 10^{-4}$ \\
\hline \multirow[t]{2}{*}{ Frontal cortex } & ZNF76 & 6 & -59.49 & $2.2 \times 10^{-6}$ \\
\hline & $H L A-G$ & 6 & 0.59 & $1.5 \times 10^{-4}$ \\
\hline \multirow[t]{2}{*}{ Hippocampus } & C11orf68 & 11 & -2.45 & $5.3 \times 10^{-8}$ \\
\hline & ZNF555 & 19 & 42.89 & $7.6 \times 10^{-6}$ \\
\hline \multirow[t]{2}{*}{ Hypothalamus } & $I K B K E$ & 1 & 0.10 & $2.7 \times 10^{-7}$ \\
\hline & $A D R B K 1$ & 11 & 4.63 & $2.4 \times 10^{-6}$ \\
\hline \multirow[t]{2}{*}{ Nucleus accumbens } & SHANK3 & 22 & 4.80 & $2.9 \times 10^{-7}$ \\
\hline & MSX2 & 5 & -4.92 & $1.2 \times 10^{-4}$ \\
\hline \multirow[t]{2}{*}{ Putamen } & $U B L 4 B$ & 1 & 5.62 & $1.0 \times 10^{-5}$ \\
\hline & SMIM6 & 17 & -1.49 & $1.0 \times 10^{-5}$ \\
\hline
\end{tabular}

Logistic regression analysis involved 820 total participants, which included 167 grade 2 or greater CNS event cases and 653 efavirenz-tolerant controls. The analysis controlled for CYP2B6/CYP2A6 genotype level, age, sex, and the first 2 principal components. The analyses included all evaluable participants without stratification for race/ancestry. The two lowest $P$-value results are shown for each brain region. CNS, central nervous system. regression analysis involving 1354 participants evaluable for baseline plasma bilirubin, and controlling for CYP2B6/ CYP2A6 genotype, age, sex, and two principal components, hepatic UGT1A1 expression was associated with bilirubin concentration $\left(P=3.4 \times 10^{-27}\right)$. This association was also present among White participants $\left(P=4.3 \times 10^{-15}\right)$, Black participants $\left(P=4.2 \times 10^{-12}\right)$, and Hispanic participants $\left(P=1.5 \times 10^{-6}\right)$ analyzed separately without adjusting for principal components (Table 4). In contrast, for 17 nonliver tissues for which PrediXcan could predict UGT1A1 expression levels, only three had nominal $P$ values less than 0.05 for association of UGT1A1 expression with baseline total bilirubin - skeletal muscle $(P=0.002)$, non-sun-exposed skin $(P=0.005)$, and putamen $(P=0.046) . P$ values exceeded 0.10 for the other 14 tissues. This established that PrediXcan could identify a true gene expression-phenotype association in our dataset, and could do so in a tissue-specific manner.

\section{Neurotransmitter transporter/receptor gene polymorphisms and CNS adverse events}

Primary analyses characterized associations between polymorphisms in the eight neurotransmitter transporter/ receptor genes $( \pm 100 \mathrm{kB})$ noted above $(S L C 6 A 2, S L C 6 A 3$, NR3C3, HTR2A, HTR2B, HTR2C, HTR6, NR3C4) and grade 2 or greater CNS adverse events within 48 weeks. Analysis among all participants, and controlling for CYP2B6/CYP2A6 genotype level, age, sex, and two principal components, the lowest nominal $P$ value was for rs12393326 in HTR2C $\left(P=6.7 \times 10^{-4}\right)$. The two lowest nominal $P$-value polymorphisms among all participants and among White, Black, and Hispanic participants analyzed separately are presented in Table 5 . None were significant after correcting for multiple testing.

\section{Genome-wide polymorphisms and CNS adverse events}

To complement the above analyses focused on eight neurotransmitter transporter/receptor gene polymorphisms, secondary analyses explored polymorphism associations genome-wide. Analysis among all participants, and controlling for CYP2B6/CYP2A6 genotype level, age,

Table 4 Associations between baseline plasma total bilirubin concentration and predicted gene expression levels in liver

\begin{tabular}{llccrc}
\hline $\begin{array}{l}\text { Race/ } \\
\text { ethnicity }\end{array}$ & Gene & Chromosome & Participants & \multicolumn{1}{c}{$\beta$} & $P$ value \\
\hline \multirow{2}{*}{ All } & UGT1A1 & 14 & 1354 & 0.30 & $3.4 \times 10^{-27}$ \\
& GGACT & 2 & 1354 & -0.88 & $7.2 \times 10^{-6}$ \\
White & UGT1A1 & 2 & 645 & 0.32 & $4.9 \times 10^{-15}$ \\
& CSN1S1 & 4 & 645 & -0.37 & $8.7 \times 10^{-5}$ \\
Black & UGT1A1 & 2 & 458 & 0.30 & $4.2 \times 10^{-12}$ \\
& PGBD2 & 1 & 458 & -1.96 & $3.6 \times 10^{-5}$ \\
Hispanic & CLDN12 & 7 & 282 & -4.17 & $3.7 \times 10^{-7}$ \\
& UGT1A1 & 2 & 282 & 0.33 & $1.5 \times 10^{-6}$ \\
\hline
\end{tabular}

Linear regression analysis involved numbers of participants shown, for baseline plasma total bilirubin concentration. Analysis among all participants controlled for CYP2B6/CYP2A6 genotype level, age, and sex. Analysis among White, Black, and Hispanic participants controlled for the same covariates but did not control for principal components. The two lowest $P$-value results are shown for each population group. 
Table 5 Associations between polymorphisms in eight neurotransmitter/transporter genes and grade 2 or greater central nervous system adverse events within $\mathbf{4 8}$ weeks of starting efavirenz

\begin{tabular}{|c|c|c|c|c|c|c|c|c|c|}
\hline Race/ethnicity & Polymorphism & Gene & Chromosome & MAF & Cases & Controls & $\beta$ & SE & $P$ value \\
\hline \multirow[t]{2}{*}{ All } & rs12393326 & HTR2C & $\mathrm{X}$ & $\mathrm{G}: 0.07$ & 164 & 815 & 0.53 & 0.16 & $6.7 \times 10^{-4}$ \\
\hline & rs5988149 & HTR2C & $x$ & $A: 0.12$ & 165 & 816 & 0.38 & 0.13 & $3.6 \times 10^{-3}$ \\
\hline \multirow[t]{2}{*}{ White } & rs660541 & PGR & 11 & $A: 0.51$ & 83 & 281 & 0.41 & 0.18 & $2.2 \times 10^{-2}$ \\
\hline & rs511484 & $P G R$ & 11 & C:0.49 & 83 & 281 & -0.36 & 0.16 & $2.3 \times 10^{-2}$ \\
\hline \multirow[t]{2}{*}{ Black } & rs2497509 & HTR2C & $x$ & C:0.43 & 49 & 211 & 0.59 & 0.18 & $8.8 \times 10^{-4}$ \\
\hline & rs2497510 & HTR2C & $x$ & $A: 0.43$ & 49 & 211 & 0.59 & 0.18 & $8.8 \times 10^{-4}$ \\
\hline \multirow[t]{2}{*}{ Hispanic } & rs3785152 & SLC6A2 & 16 & $\mathrm{~T}: 0.08$ & 26 & 158 & 1.09 & 0.46 & $1.8 \times 10^{-2}$ \\
\hline & rs2016711 & HTR2A & 13 & G:0.03 & 26 & 158 & 1.34 & 0.61 & $2.8 \times 10^{-2}$ \\
\hline
\end{tabular}

Logistic regression analysis involved the indicated numbers of grade 2 or greater CNS event cases and efavirenz-tolerant controls. We considered polymorphisms in SLC6A2, SLC6A3, NR3C3, HTR2A, HTR2B, HTR2C, HTR6, and NR3C4 $\pm 100 \mathrm{kB}$. The analysis controlled for CYP2B6/CYP2A6 genotype level, age, sex, and, for the analysis involving all participants, the first two principal components. The two lowest $P$-value results are shown for each race/ancestry group.

CNS, central nervous system; MAF, minor allele frequency.

Table 6 Associations between genome-wide polymorphisms and grade 2 or greater central nervous system adverse events within 48 weeks of starting efavirenz

\begin{tabular}{|c|c|c|c|c|c|c|c|c|c|}
\hline Race/ethnicity & Polymorphism & Gene & Chromosome & MAF & Cases & Controls & $\beta$ & SE & $P$ value \\
\hline \multirow[t]{2}{*}{ All } & rs7143465 & SLC8A3 & 14 & C:0.45 & 167 & 653 & -0.69 & 0.12 & $2.2 \times 10^{-9}$ \\
\hline & rs1045920 & CFAP36 & 2 & $\mathrm{~T}: 0.45$ & 166 & 653 & -0.74 & 0.13 & $2.6 \times 10^{-9}$ \\
\hline \multirow[t]{2}{*}{ Black } & rs2292493 & $B B S 12$ & 4 & $\mathrm{~T}: 0.13$ & 52 & 211 & 1.60 & 0.31 & $2.9 \times 10^{-7}$ \\
\hline & rs72671080 & BBS12 & 4 & $A: 0.13$ & 52 & 211 & 1.60 & 0.31 & $3.0 \times 10^{-7}$ \\
\hline \multirow[t]{2}{*}{ Hispanic } & rs62520409 & LOC105375706 & 8 & $A: 0.04$ & 26 & 158 & 3.34 & 0.68 & $9.8 \times 10^{-7}$ \\
\hline & rs1305010 & Intergenic & 2 & C:0.06 & 26 & 157 & 2.31 & 0.54 & $1.9 \times 10^{-5}$ \\
\hline \multirow[t]{2}{*}{ White } & rs10823547 & Intergenic & 10 & G:0.50 & 82 & 281 & -0.88 & 0.18 & $1.2 \times 10^{-6}$ \\
\hline & rs144103499 & ACSF3 & 16 & CCT:0.21 & 83 & 280 & 1.04 & 0.22 & $1.3 \times 10^{-6}$ \\
\hline
\end{tabular}

Logistic regression analysis involved the indicated numbers of grade 2 or greater CNS event cases and efavirenz-tolerant controls. The analysis controlled for CYP2B6/ CYP2A6 genotype level, age, sex, and, for the analysis involving all participants, the first two principal components. The two lowest $P$-value results are shown for each race/ ancestry group.

CNS, central nervous system; MAF, minor allele frequency.

sex, and two principal components, the lowest nominal $P$ value was for rs7143465 in SLC8A3, which encodes solute carrier family 8 member A3 $\left(P=2.2 \times 10^{-9}\right)$. The two lowest nominal $P$-value polymorphisms among all participants and among White, Black, and Hispanic participants analyzed separately are presented in Table 6 .

For the four genes in Table 6, we attempted to examine whether predicted expression in brain was associated with CNS events. Of these, CFAP36 was not represented in PrediXcan. Among White participants, but not among Black or Hispanic participants, predicted ACSF3 expression in brain tissues tended to be associated with grade 2 or greater CNS adverse events within 48 weeks, including in anterior cingulate cortex $\left(P=9.46 \times 10^{-6}\right)$, frontal cortex $\left(P=1.08 \times 10^{-5}\right)$, cortex $\left(1.24 \times 10^{-5}\right)$, and caudate $\left(P=8.53 \times 10^{-5}\right)$. Of note, ACSF3 rs144103499 (Table 6) is not a known expression quantitative trait locus for ACSF3 in any tissue [42]. There were not associations with BBS12 or SLC8A3. The lowest $P$ value for $B B S 12$ was in cerebellum among Black participants $(P=0.092)$, and for SLC8A3 was in hypothalamus among all participants $(P=0.014)$.

\section{Discussion}

Among individuals who were randomly assigned to initial treatment with efavirenz-containing regimens in four ACTG studies, and with correction for multiple comparisons, we found no significant association between predicted expression of six neurotransmitter transporter and receptor genes postulated to mediate efavirenz effects in brain (SLC6A2, SLC6A3, PGR, HTR2A, HTR2B, HTR6) and grade 2 or greater CNS adverse events, both among all participants, and in analyses performed separately among White, Black, and Hispanic participants. The lowest nominal $P$ value among all participants was for $P G R$ in hippocampus $(P=0.012)$. Similarly, after correction for multiple comparisons we found no significant association between individuals SNPs in these genes or $A R$ and $H T R 2 C$ and grade 2 or greater CNS adverse events, both among all participants, and in analyses performed separately among White, Black, and Hispanic participants. The lowest nominal $P$ value was for rs12393326 in HTR2C $\left(P=6.7 \times 10^{-4}\right)$.

Analyses were controlled for CYP2B6/CYP2A6 genotype, age, and sex, and, in analyses that pooled all race/ethnicity groups, also the first two principal components. We demonstrated significant, though weak, associations between CYP2B6/CYP2A6 genotype level and grade 2 or greater CNS adverse events, which is generally consistent with several previous reports $[18,23,24]$. It was therefore important that we adjust for CYP2B6/CYP2A6 genotype level in analyses for associations with predicted neurotransmitter transporter/receptor gene expression and polymorphisms. In addition, controlling for principal components in analyses involving all participants reduced 
the likelihood for false associations due to unrecognized population stratification.

PrediXcan is a relatively new computational algorithm that allows the heritable component of RNA expression levels for individual genes in different tissues to be inferred from genome-wide genotype data [25]. It was developed to exploit genotype-tissue expression data, and evaluates aggregate effects of cis-regulatory variants (within $1 \mathrm{MB}$ upstream or downstream) on gene expression by an elastic net regression method, and generates potential expression quantitative trait loci and their weights for each gene in each genotype-tissue expression tissue type. By considering genes rather than individual polymorphisms, PrediXcan should have a greatly reduced multiple testing burden versus single-variant-single-trait association tests. PrediXcan may therefore identify loci with modest to weak effect sizes that are not significant in genome-wide association studies. To assess the performance of PrediXcan we tested for associations with baseline plasma total bilirubin concentration. It is reassuring that we found significant associations between predicted UGT1A1 expression in liver and bilirubin concentrations among all participants, and separately among White, Black, and Hispanic participants. Thus, PrediXcan detected a known association in a tissuespecific manner in our dataset.

By analyzing all participants pooled, as well as racial/ ethnic groups of White, Black, and Hispanic participants separately, we had the potential to identify consistent genetic associations across groups. Although true genetic associations need not be present in all populations, finding the same association in all participants and in each race/ethnicity group increases the likelihood that the association is not by chance. For example, associations between CYP2B6 genotype level and plasma efavirenz concentrations were previously shown in pooled analyses and among White, Black, and Hispanic participants analyzed separately [15]. In the present analyses, neither predicted gene expression levels or polymorphisms with the lowest $P$ values were consistent across populations.

We cannot explain the apparent association between female sex and fewer grade 2 or greater CNS adverse events among all participants, and among White participants and Black participants analyzed separately. Previous reports have been inconsistent in this regard, with studies showing no difference by sex $[43,44]$, increased efavirenz CNS adverse events in males [45], and increased CNS adverse events in females [46].

This study has limitations. Because providers may not have referred patients perceived to be at increased risk for CNS adverse events to studies of efavirenz, risk may be underestimated. Analyses largely involved White, Black, and Hispanic participants in the USA, so findings may not translate to other countries or race/ethnicity groups.
The open-label design of three of the four studies might have biased investigators into reporting CNS adverse events in patients randomized to efavirenz. Although PrediXcan readily identified an association between predicted UGT1A1 expression in liver and bilirubin concentrations, this does not prove that we could detect associations of efavirenz CNS adverse events with predicted gene expression levels in brain. To our knowledge, there is no brain gene-phenotype pair in our dataset that could serve as a positive control. A larger sample size would increase power to identify associations. The present study was not designed to address rare polymorphisms, epigenetics, inducibility of gene expression, and trans-regulatory elements. In addition, factors not evaluated in this study such as nicotine and alcohol use may affect expressions of $C Y P 2 B 6, C Y P 2 A 6$, and other genes, and could conceivably differ by ancestry.

\section{Conclusion}

It is important to identify genetic factors that affect susceptibility to antiretroviral toxicities. The present study suggests that interindividual differences in brain neurotransmitter transporter/receptor genomics may not explain variable susceptibility to efavirenz CNS adverse events.

\section{Acknowledgements}

The authors are grateful to the many persons with HIV infection who volunteered for ACTG384, A5095, A5142, A5202, and A5128. In addition, the authors acknowledge the contributions of study teams and site staff for these protocols.

Research reported in this publication was supported by the National Institute of Allergy and Infectious Diseases of the National Institutes of Health under Award Number UM1 AI068634, UM1 AI068636, and UM1 AI106701. This work was supported by the AIDS Clinical Trials Group funded by the National Institute of Allergy and Infectious Diseases (AI068636, AI038858, AI068634, AI038855). Grant support included AI069439, TR000445 (DWH), AI077505 (DWH), and AI50410 (University of North Carolina at Chapel Hill Center for AIDS Research). Clinical Research Sites that participated in ACTG protocols ACTG384, A5095, A5142, and A5202, and collected DNA under protocol A5128, were supported by the following grants from NIAID: AI069477, AI027675, AI073961, AI069474, AI069432, AI069513, AI069423, AI050410, AI069452, AI69450, AI054907, AI069428, AI045008, AI069495, AI069415, AI069556, AI069484, AI069424, AI069532, AI069419, AI069471, AI025859, AI069418, AI050409, AI069501, AI069502, AI069511, AI069434, AI069465, AI069494, AI069472, AI069470, AI046376, AI072626, AI027661, AI034853, AI069447, AI032782, AI027658, AI27666, AI058740, AI046370, and by the following grants from the National Center for Research Resources (NCRR): RR00051, RR00046, RR025747, RR025777, RR024160, 
RR024996, and RR024156. Abbott Laboratories, BoehringerIngelheim, Bristol-Myers Squibb, Gilead Sciences, and GlaxoSmithKline provided study medications.

\section{Conflicts of interest}

Joseph J. Eron has received research support from grants awarded to UNC from BMS, AbbVie, Janssen, ViiV Healthcare, and Gilead. He is a consultant to BMS, AbbVie, Janssen, ViiV Healthcare, Merck, and Gilead. Paul E. Sax has received research support from grants awarded to Brigham and Women's Hospital from BMS, ViiV, and Gilead. He is a consultant to BMS, AbbVie, Janssen, ViiV, Merck, and Gilead. Eric S. Daar has received research support from grants awarded to Los Angeles Biomedical Research Institute at Harbor-UCLA Medical Center from Gilead, Merck and ViiV. He is a consultant for Bristol-Myers Squibb, Janssen, Merck, Teva, and ViiV. Sharon A. Riddler has received research support from grants awarded to University of Pittsburgh from Gilead Sciences and Bristol-Myers Squibb. For the remaining authors, there are no conflicts of interest.

\section{References}

1 van Leth F, Phanuphak P, Ruxrungtham K, Baraldi E, Miller S, Gazzard B, et al. Comparison of first-line antiretroviral therapy with regimens including nevirapine, efavirenz, or both drugs, plus stavudine and lamivudine: a randomised open-label trial, the 2NN Study. Lancet 2004; 363:1253-1263.

2 Gulick RM, Ribaudo HJ, Shikuma CM, Lustgarten S, Squires KE, Meyer WA III, et al. Triple-nucleoside regimens versus efavirenz-containing regimens for the initial treatment of HIV-1 infection. N Engl J Med 2004; 350:1850-1861.

3 Riddler SA, Haubrich R, DiRienzo AG, Peeples L, Powderly WG, Klingman $\mathrm{KL}$, et al. Class-sparing regimens for initial treatment of HIV-1 infection. N Engl J Med 2008; 358:2095-2106.

4 Lennox JL, DeJesus E, Lazzarin A, Pollard RB, Madruga JV, Berger DS, et al. Safety and efficacy of raltegravir-based versus efavirenz-based combination therapy in treatment-naive patients with HIV-1 infection: a multicentre, double-blind randomised controlled trial. Lancet 2009; 374:796-806.

5 Cooper DA, Heera J, Goodrich J, Tawadrous M, Saag M, Dejesus E, et al. Maraviroc versus Efavirenz, both in combination with zidovudine-lamivudine, for the treatment of antiretroviral-naive subjects with CCR5-tropic HIV-1 infection. J Infect Dis 2010; 201:803-813.

6 Daar ES, Tierney C, Fischl MA, Sax PE, Mollan K, Budhathoki C, et al. Atazanavir plus ritonavir or efavirenz as part of a 3-drug regimen for initial treatment of HIV-1. Ann Intern Med 2011; 154:445-456.

7 Clifford DB, Evans S, Yang Y, Acosta EP, Goodkin K, Tashima K, et al. Impact of efavirenz on neuropsychological performance and symptoms in HIVinfected individuals. Ann Intern Med 2005; 143:714-721.

8 Mollan KR, Smurzynski M, Eron JJ, Daar ES, Campbell TB, Sax PE, et al. Association between efavirenz as initial therapy for HIV-1 infection and increased risk for suicidal ideation or attempted or completed suicide: an analysis of trial data. Ann Intern Med 2014; 161:1-10.

9 Arenas-Pinto A, Grund B, Sharma S, Martinez E, Cummins N, Fox J, et al. Risk of suicidal behavior with use of efavirenz: results from the strategic timing of antiretroviral treatment trial. Clin Infect Dis 2018. [Epub ahead of print].

10 Haas DW, Ribaudo HJ, Kim RB, Tierney C, Wilkinson GR, Gulick RM, et al. Pharmacogenetics of efavirenz and central nervous system side effects: an Adult AIDS Clinical Trials Group study. AIDS 2004; 18:2391-2400.

11 Tsuchiya K, Gatanaga H, Tachikawa N, Teruya K, Kikuchi Y, Yoshino M, et al. Homozygous CYP2B6 *6 (Q172H and K262R) correlates with high plasma efavirenz concentrations in HIV-1 patients treated with standard efavirenzcontaining regimens. Biochem Biophys Res Commun 2004; 319:1322-1326.

12 Rotger M, Colombo S, Furrer H, Bleiber G, Buclin T, Lee BL, et al. Influence of CYP2B6 polymorphism on plasma and intracellular concentrations and toxicity of efavirenz and nevirapine in HIV-infected patients. Pharmacogenet Genom 2005; 15:1-5.
13 Haas DW, Smeaton LM, Shafer RW, Robbins GK, Morse GD, Labbe L, et al. Pharmacogenetics of long-term responses to antiretroviral regimens containing efavirenz and/or nelfinavir: an Adult AIDS Clinical Trials Group Study. J Infect Dis 2005; 192:1931-1942.

14 Rodriguez-Novoa S, Barreiro P, Rendon A, Jimenez-Nacher I, GonzalezLahoz J, Soriano V. Influence of $516 \mathrm{G}>\mathrm{T}$ polymorphisms at the gene encoding the CYP450-2B6 isoenzyme on efavirenz plasma concentrations in HIV-infected subjects. Clin Infect Dis 2005; 40:1358-1361.

15 Holzinger ER, Grady B, Ritchie MD, Ribaudo HJ, Acosta EP, Morse GD, et al. Genome-wide association study of plasma efavirenz pharmacokinetics in AIDS Clinical Trials Group protocols implicates several CYP2B6 variants. Pharmacogenet Genom 2012; 22:858-867.

16 Wyen C, Hendra H, Vogel M, Hoffmann C, Knechten H, Brockmeyer NH, et al. Impact of CYP2B6 983T > C polymorphism on non-nucleoside reverse transcriptase inhibitor plasma concentrations in HIV-infected patients. J Antimicrob Chemo 2008; 61:914-918.

17 Wang J, Sonnerborg A, Rane A, Josephson F, Lundgren S, Stahle L, et al. Identification of a novel specific CYP2B6 allele in Africans causing impaired metabolism of the HIV drug efavirenz. Pharmacogenet Genom 2006; 16:191-198.

18 Ribaudo HJ, Liu H, Schwab M, Schaeffeler E, Eichelbaum M, MotsingerReif AA, et al. Effect of CYP2B6, ABCB1, and CYP3A5 polymorphisms on efavirenz pharmacokinetics and treatment response: an AIDS Clinical Trials Group study. J Infect Dis 2010; 202:717-722.

19 di lulio J, Fayet A, Arab-Alameddine M, Rotger M, Lubomirov R, Cavassini M, et al. In vivo analysis of efavirenz metabolism in individuals with impaired CYP2A6 function. Pharmacogenet Genom 2009; 19:300-309.

20 Kwara A, Lartey M, Sagoe KW, Rzek NL, Court MH. CYP2B6 (c.516G- > T) and CYP2A6 ( ${ }^{*} 9 \mathrm{~B}$ and/or *17) polymorphisms are independent predictors of efavirenz plasma concentrations in HIV-infected patients. $\mathrm{Br} J \mathrm{Clin}$ Pharmacol 2009; 67:427-436.

21 Kwara A, Lartey M, Sagoe KW, Kenu E, Court MH. CYP2B6, CYP2A6 and UGT2B7 genetic polymorphisms are predictors of efavirenz mid-dose concentration in HIV-infected patients. AIDS 2009; 23:2101-2106.

22 Haas DW, Kwara A, Richardson DM, Baker P, Papageorgiou I, Acosta EP, et al. Secondary metabolism pathway polymorphisms and plasma efavirenz concentrations in HIV-infected adults with CYP2B6 slow metabolizer genotypes. J Antimicrob Chemother 2014; 69:2175-2182.

23 Leger $\mathrm{P}$, Chirwa S, Turner M, Richardson DM, Baker P, Leonard M, et al. Pharmacogenetics of efavirenz discontinuation for reported central nervous system symptoms appears to differ by race. Pharmacogenet Genomics 2016; 26:473-480.

24 Mollan KR, Tierney C, Hellwege JN, Eron JJ, Hudgens MG, Gulick RM, et al. Race/Ethnicity and the pharmacogenetics of reported suicidality with efavirenz among clinical trials participants. J Infect Dis 2017; 216:554-564.

25 Gamazon ER, Wheeler HE, Shah KP, Mozaffari SV, Aquino-Michaels K, Carroll RJ, et al. A gene-based association method for mapping traits using reference transcriptome data. Nat Genet 2015; 47:1091-1098.

26 Robbins GK, De GV, Shafer RW, Smeaton LM, Snyder SW, Pettinelli C, et al. Comparison of sequential three-drug regimens as initial therapy for HIV1 infection. N Engl J Med 2003; 349:2293-2303.

27 Shafer RW, Smeaton LM, Robbins GK, De GV, Snyder SW, D'Aquila RT, et al. Comparison of four-drug regimens and pairs of sequential three-drug regimens as initial therapy for HIV-1 infection. N Engl J Med 2003; 349:2304-2315.

28 Gulick RM, Ribaudo HJ, Shikuma CM, Lalama C, Schackman BR, Meyer WA III, et al. Three- vs four-drug antiretroviral regimens for the initial treatment of HIV-1 infection: a randomized controlled trial. JAMA 2006; 296:769-781.

29 Haas DW, Wilkinson GR, Kuritzkes DR, Richman DD, Nicotera J, Mahon LF, et al. A multi-investigator/institutional DNA bank for AIDS-related human genetic studies: AACTG Protocol A5128. HIV Clin Trials 2003; 4:287-300.

30 US Department of Health and Human Services; National Institutes of Health; National Institute of Allergy and Infectious Diseases; Division of AIDS. Division of AIDS table for grading the severity of adult and pediatric adverse events, version 1.0; 2009. Available at: $h t t p: / / r s c . t e c h-r e s . c o m / d o c s / d e f a u l t-$ source/safety/table_for_grading_severity_of_adult_pediatric_adverse_ events.pdf. [Accessed 28 April 2018].

31 Pereyra F, Jia X, McLaren PJ, Telenti A, de Bakker PI, Walker BD, et al. The major genetic determinants of HIV-1 control affect HLA class I peptide presentation. Science 2010; 330:1551-1557.

32 Moore CB, Verma A, Pendergrass S, Verma SS, Johnson DH, Daar ES, et al. Phenome-wide association study relating pretreatment laboratory parameters with human genetic variants in AIDS Clinical Trials Group Protocols. Open Forum Infect Dis 2015; 2:ofu113. 
33 Purcell S, Neale B, Todd-Brown K, Thomas L, Ferreira MA, Bender D, et al. PLINK: a tool set for whole-genome association and population-based linkage analyses. Am J Hum Genet 2007; 81:559-575.

34 Team RDC. R: a language and environment for statistical computing. Vienna, Austria: R Foundation for Statistical Computing; 2011.

35 Genomes Project C, Abecasis GR, Altshuler D, Auton A, Brooks LD, Durbin RM, et al. A map of human genome variation from population-scale sequencing. Nature 2010; 467:1061-1073.

36 liftOver. Lift Genome Annotations. Available at: http://genome.ucsc.edu/cgibin/hgLiftOver. [Accessed 28 April 2018].

37 Delaneau O, Zagury JF, Marchini J. Improved whole-chromosome phasing for disease and population genetic studies. Nat Methods 2013; 10:5-6.

38 Howie BN, Donnelly P, Marchini J. A flexible and accurate genotype imputation method for the next generation of genome-wide association studies. PLoS Genet 2009; 5:e1000529.

39 Verma SS, de Andrade M, Tromp G, Kuivaniemi H, Pugh E, Namjou B, et al. Imputation and quality control steps for combining multiple genome-wide datasets. Front Genet 2014; 5:370.

40 Price A. EIGENSOFT. Available at: http://www.hsph.harvard.edu/alkesprice/software/. [Accessed 28 April 2018].
41 Li B, Verma SS, Veturi YC, Verma A, Bradford Y, Haas DW, et al. Evaluation of PrediXcan for prioritizing GWAS associations and predicting gene expression. Pac Symp Biocomput 2018; 23:448-459.

42 GTEx. The GTEx Project; 2018. Available at: https://www.gtexportal.org/ home/documentationPage. [Accessed 22 April 2018].

43 Wynberg E, Williams E, Tudor-Williams G, Lyall H, Foster C. Discontinuation of efavirenz in paediatric patients: why do children switch? Clin Drug Investig 2018; 38:231-238.

44 Smith KY, Tierney C, Mollan K, Venuto CS, Budhathoki C, Ma Q, et al. Outcomes by sex following treatment initiation with atazanavir plus ritonavir or efavirenz with abacavir/lamivudine or tenofovir/emtricitabine. Clin Infect Dis 2014; 58:555-563.

45 Dhoro M, Ngara B, Kadzirange G, Nhachi C, Masimirembwa C. Genetic variants of drug metabolizing enzymes and drug transporter (ABCB1) as possible biomarkers for adverse drug reactions in an HIVIAIDS cohort in Zimbabwe. Curr HIV Res 2013; 11:481-490.

46 Bisaso KR, Mukonzo JK, Ette El. Markov model for characterizing neuropsychologic impairment and Monte Carlo simulation for optimizing efavirenz therapy. J Clin Pharmacol 2015; 55:1229-1235. 